\title{
Le dossier électronique du patient traverse une phase critique
}

\section{Yvonne Gilli}

Dr, membre du Comité central de la FMH, responsable du département Numérisation / eHealth

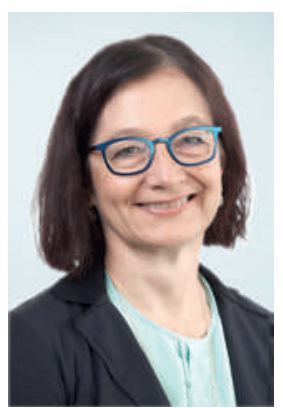

L'Office fédéral de la santé publique (OFSP) et eHealth Suisse en sa qualité d'organe de coordination entre la Confédération et les cantons travaillent actuellement activement aux dispositions d'exécution de la loi sur le dossier électronique du patient (LDEP). Le calendrier est ambitieux: les ordonnances d'application devraient en effet être terminées au plus tard au premier trimestre 2017. La loi sur le dossier électronique du patient pourra être mise en vigueur dès cette date.

Le délai pour les prises de position sur le premier projet du droit d'exécution est à présent écoulé. A cette occasion, la FMH a pris position de manière détaillée et résolument critique. Toutes les prises de position ont été publiées sur le site Internet de l'OFSP où elles peuvent être consultées. Ce que nous craignions s'est produit: la densité normative et les exigences sont trop élevées. La profusion de détails techniques et juridiques rend les dispositions d'exécution inadéquates et difficilement lisibles. Pour les médecins, le dossier électronique du patient ne s'imposera que si les procédures nécessaires au quotidien sont clarifiées et prises en compte, ce qui n'est pas le cas à présent.

La densité normative et les exigences du dossier électronique du patient sont trop élevées.

Les responsables de l'OFSP ont pris au sérieux les critiques largement partagées et sont prêts à remanier des points importants de l'ordonnance. Pour garantir le lien avec la pratique et le bénéfice pour les patients et les professionnels de la santé, les associations professionnelles concernées doivent à présent unir leurs forces. Regroupant huit associations nationales (ChiroSuisse, les ergothérapeutes, les diététiciens, la FMH, les sages-femmes, pharmaSuisse, physiosuisse et l'ASI), le groupe de travail interprofessionnel dédié au dossier électronique du patient (IPAG EPD) joue ici un rôle clé. L'IPAG est soumis à une énorme pression. Cette large collaboration interprofessionnelle n'existe que depuis deux ans; or développer une culture de la communication commune et une responsabilité solidaire requiert du temps et la liberté de pouvoir se consacrer dans un premier temps à de petits champs d'action communs. Deux conditions dont nous ne disposons pas pour l'élaboration du dossier électronique du patient.

L'IPAG a dû livrer de premiers résultats à eHealth Suisse et à l'OFSP cet été déjà, ce qui a représenté un véritable défi! Toutes les associations concernées ont dû mobiliser des ressources dépassant leurs capacités usuelles. L'IPAG est néanmoins parvenu à adopter un rapport consensuel illustrant les procédures thérapeutiques interprofessionnelles en se fondant sur l'exemple de la cybermédication et sur un exemple de cas.

\section{Il est essentiel de se concentrer sur} des points centraux qui doivent être définis avec les fournisseurs de prestations.

La cybermédication est un système d'information électronique sur le processus de médication. Certains de ses aspects sont d'ores et déjà standardisés et bien implantés au niveau international, c'est pourquoi elle constitue un des premiers éléments à reprendre dans le dossier électronique du patient. Pour l'IPAG, les principaux éléments de la cybermédication sont les suivants: vue d'ensemble de la médication actuelle, ordonnance électronique, informations concernant la remise et l'utilisation de médicaments et possibilité d'ajouter un commentaire (texte libre).

Ce que nous attendons à présent de l'OFSP, c'est un ralentissement du processus de mise en œuvre et la concentration sur quelques points centraux qui ne peuvent être définis qu'avec les acteurs concernés. Les détails techniques ne peuvent pas faire l'objet d'une ordonnance; il s'agit d'éléments statiques et à ce titre, ils ne feraient qu'entraver l'évolution dynamique du dossier. Si ces conditions sont réunies, la FMH voit le DEP comme une chance et elle se réjouit de continuer de participer à son développement. 